Acta Crystallographica Section E

Structure Reports

Online

ISSN 1600-5368

\section{6-Chloro-4-(2-phenylethenyl)chroman-2- one}

\section{Kwang-Su Choi and Sung-Gon Kim*}

Department of Chemistry, Kyonggi University, San 94-6, lui-dong, Yeongtong-gu, Suwon 443-760, Republic of Korea

Correspondence e-mail: sgkim123@kgu.ac.kr

Received 2 November 2010; accepted 3 November 2010

Key indicators: single-crystal X-ray study; $T=100 \mathrm{~K}$; mean $\sigma(\mathrm{C}-\mathrm{C})=0.002 \AA$; $R$ factor $=0.033 ; w R$ factor $=0.083 ;$ data-to-parameter ratio $=18.4$.

The title compound, $\mathrm{C}_{17} \mathrm{H}_{13} \mathrm{ClO}_{2}$, was obtained from the oxidation of 6-chloro-4-(2-phenylethenyl)chroman-2-ol, which was synthesized by the reaction of of $(E)-3-(5-c h l o r o-2-$ hydroxyphenyl)acrylaldehyde with styrylboronic acid using diethylamine as a catalyst. The six-membered pyranone ring of the chromane system has a screw-boat conformation. The dihedral angle between the least-squares planes of the chromane ring system and the styryl group is $85.28(9)^{\circ}$.

\section{Related literature}

For the synthesis of the title compound, see: Choi \& Kim (2010). For the biological activity of chromenes, see: Ellis \& Lockhart (2007); Green et al. (1996); Horton et al. (2003).<smiles>O=C1CC(/C=C/c2ccccc2)c2cc(Cl)ccc2O1</smiles>

\section{Experimental}

Crystal data

$\mathrm{C}_{17} \mathrm{H}_{13} \mathrm{ClO}_{2}$

Monoclinic, $P 2_{1} / c$

$a=15.6682(3) \AA$

$b=6.2800(1) \AA$

$c=14.9383(3) \AA$

$\beta=115.129(1)^{\circ}$

$$
\begin{aligned}
& V=1330.76(4) \AA^{3} \\
& Z=4 \\
& \text { Mo } K \alpha \text { radiation } \\
& \mu=0.29 \mathrm{~mm}^{-1} \\
& T=100 \mathrm{~K} \\
& 0.28 \times 0.13 \times 0.05 \mathrm{~mm}
\end{aligned}
$$

\section{Data collection}

Bruker APEXII CCD

diffractometer

Absorption correction: multi-scan (SADABS; Bruker, 2005)

$T_{\min }=0.925, T_{\max }=0.986$

Refinement

$R\left[F^{2}>2 \sigma\left(F^{2}\right)\right]=0.033$

$w R\left(F^{2}\right)=0.083$

$S=1.06$

3325 reflections

12258 measured reflections 3325 independent reflections 2839 reflections with $I>2 \sigma(I)$ $R_{\text {int }}=0.021$

Data collection: APEX2 (Bruker, 2005); cell refinement: SAINT (Bruker, 2005); data reduction: $S A I N T$; $\operatorname{program}(\mathrm{s})$ used to solve structure: SHELXS97 (Sheldrick, 2008); program(s) used to refine structure: SHELXL97 (Sheldrick, 2008); molecular graphics: SHELXTL (Sheldrick, 2008); software used to prepare material for publication: SHELXTL.

This research was supported by the Basic Science Research Program through the National Research Foundation of Korea (NRF) funded by the Ministry of Education, Science and Technology (2010-0004139).

Supplementary data and figures for this paper are available from the IUCr electronic archives (Reference: IS2627).

\section{References}

Bruker (2005). APEX2, SAINT and SADABS. Bruker AXS Inc., Madison, Wisconsin, USA.

Choi, K.-S. \& Kim, S.-G. (2010). Tetrahedron Lett. 51, 5203-5206.

Ellis, G. P. \& Lockhart, I. M. (2007). The Chemistry of Heterocyclic Compounds, Chromenes, Chromanones, and Chromones, Vol. 31, edited by G. P. Ellis, pp. 1-119. New York: Wiley-VCH.

Geen, G. R., Evans, J. M. \& Vong, A. K. (1996). Comprehensive Heterocyclic Chemistry II: Pyrans and their Benzo Derivatives: Applications, Vol. 5, edited by A. R. Katritzky, C. W. Rees \& E. F. V. Scriven, pp. 469-500. Oxford: Pergamon Press.

Horton, D. A., Boume, G. T. \& Smythe, M. L. (2003). Chem. Rev. 103, 893-930. Sheldrick, G. M. (2008). Acta Cryst. A64, 112-122. 


\section{supporting information}

Acta Cryst. (2010). E66, o3104 [https://doi.org/10.1107/S1600536810045101]

\section{6-Chloro-4-(2-phenylethenyl)chroman-2-one}

\section{Kwang-Su Choi and Sung-Gon Kim}

\section{S1. Comment}

Chromanes (dihydrobenzopyranes) are ubiquitously found in numerous biologically active natural products. Molecules containing chromane scaffolds exhibit a broad range of bioactivities, such as antiviral, antitumor, antimicrobial, sex pheromone, and those of the central nervous system activity (Ellis \& Lockhart, 2007; Green et al., 1996; Horton et al. 2003). We report herein the crystal structure of the title compound, which belongs to this class of compounds.

In the title compound, the six-membered pyranone ring of the chromane system has a screw-boat conformation. The dihedral angle between the least-squares planes of the chromane ring system and the styryl group is $85.28(9)^{\circ}$.

\section{S2. Experimental}

To a solution of triethylamine $(0.10 \mathrm{mmol})$ in $\mathrm{CH}_{2} \mathrm{Cl}_{2}(1.5 \mathrm{ml})$ was added styrylboronic acid $(0.60 \mathrm{mmol})$ at room temperature. The solution was stirred for $5 \mathrm{~min}$ before addition of (E)-3-(5-chloro-2-hydroxyphenyl)acrylaldehyde (0.50 $\mathrm{mmol})$. After stirring for $3 \mathrm{~h}$, the resulting mixture was direct purified by silica gel chromatography to afford 6chloro-3,4-dihydro-4-styryl-2 $H$-chromen-2-ol. Oxidation of 6-chloro-3,4-dihydro-4-styryl-2 $H$-chromen-2-ol (0.40 mmol) was performed in $\mathrm{CH}_{2} \mathrm{Cl}_{2}(2.0 \mathrm{ml})$ by adding of pyridinium chlorochromate $(0.40 \mathrm{mmol})$ at room temperature. After $3 \mathrm{~h}$, additional pyridinium chlorochromate $(0.40 \mathrm{mmol})$ was added and after $6 \mathrm{~h}$ purification by silica gel chromatography was afforded the title compound (Fig. 2). Crystals suitable for X-ray analysis were obtained by slow evaporation from an $n$ hexane $/ \mathrm{CH}_{2} \mathrm{Cl}_{2}$ solution.

\section{S3. Refinement}

All $\mathrm{H}$ atoms were positioned geometrically, with $\mathrm{C}-\mathrm{H}=0.93-0.98 \AA$ and constrained to ride on their parent atoms, with $U_{\text {iso }}(\mathrm{H})=x U_{\text {eq }}(\mathrm{C})$, where $x=1.5$ for methyl $\mathrm{H}$ and $x=1.2$ for all other $\mathrm{H}$ atoms. 


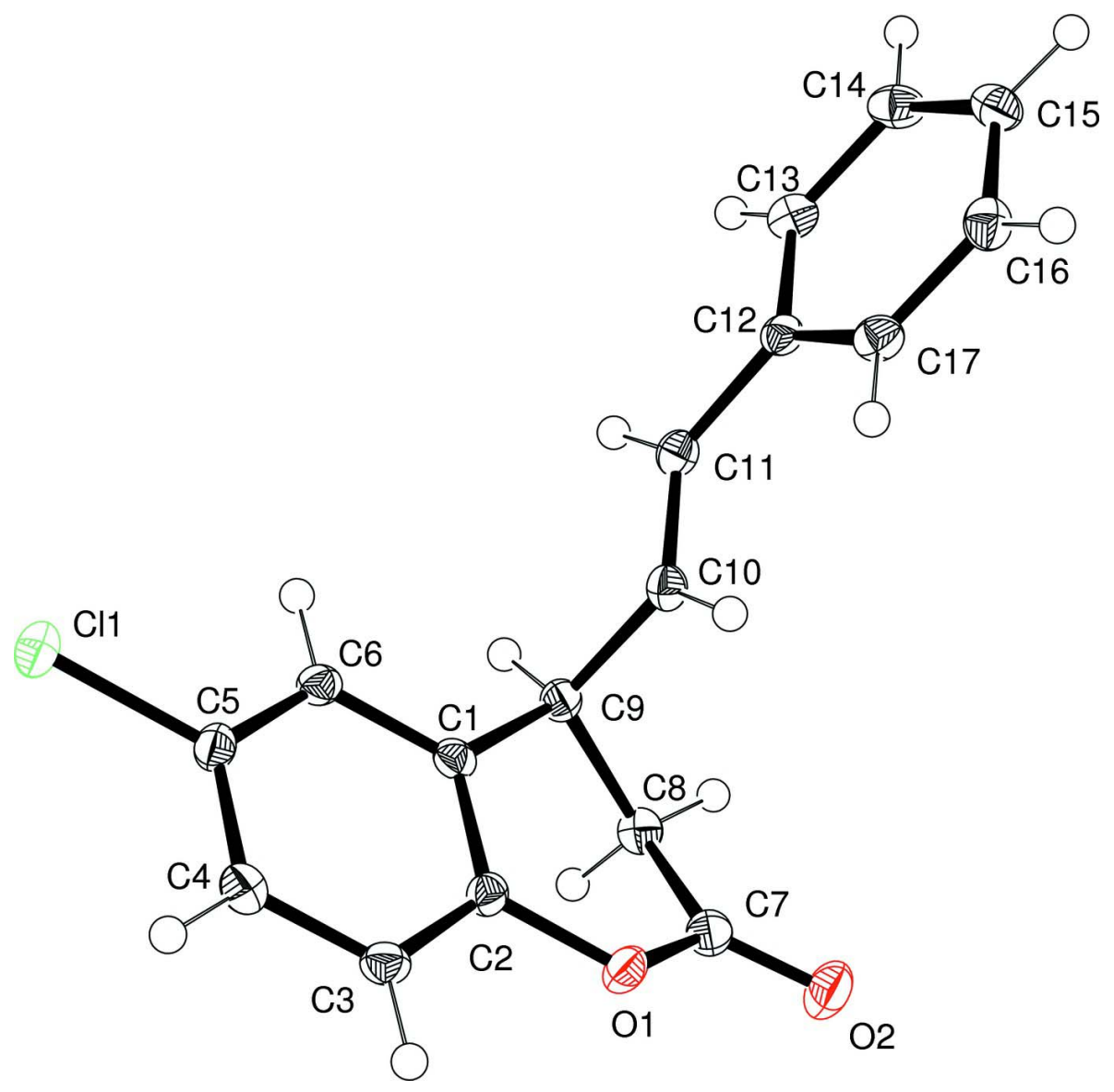

Figure 1

A view of the molecular structure of the title compound, showing the atom-numbering scheme. Displacement ellipsoids are drawn at the $50 \%$ probability level.
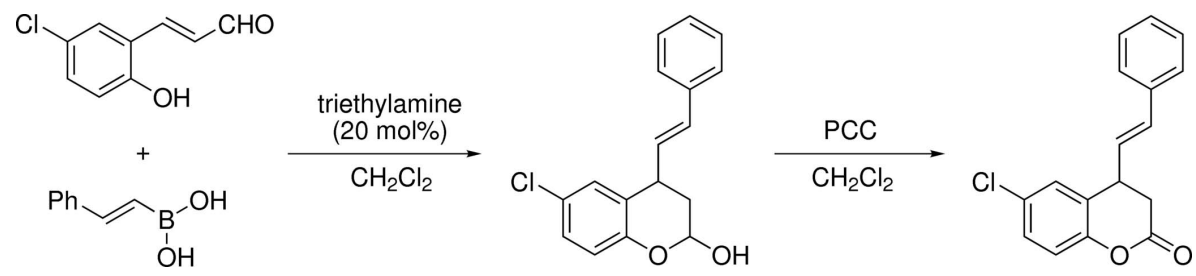

Figure 2

The preparation scheme of the title compound.

6-Chloro-4-(2-phenylethenyl)chroman-2-one

Crystal data

$\mathrm{C}_{17} \mathrm{H}_{13} \mathrm{ClO}_{2}$

$M_{r}=284.72$

Monoclinic, $P 2_{1} / c$

Hall symbol: -P $2 \mathrm{ybc}$

$a=15.6682(3) \AA$

$b=6.2800(1) \AA$

$c=14.9383(3) \AA$

$\beta=115.129(1)^{\circ}$
$V=1330.76(4) \AA^{3}$

$Z=4$

$F(000)=592$

$D_{\mathrm{x}}=1.421 \mathrm{Mg} \mathrm{m}^{-3}$

Mo $K \alpha$ radiation, $\lambda=0.71073 \AA$

Cell parameters from 5561 reflections

$\theta=3.6-28.3^{\circ}$

$\mu=0.29 \mathrm{~mm}^{-1}$ 
$T=100 \mathrm{~K}$

Block, silver

\section{Data collection}

\section{Bruker APEXII CCD} diffractometer

Radiation source: fine-focus sealed tube Graphite monochromator $\varphi$ and $\omega$ scans

Absorption correction: multi-scan (SADABS; Bruker, 2005)

$T_{\min }=0.925, T_{\max }=0.986$

\section{Refinement}

Refinement on $F^{2}$

Least-squares matrix: full

$R\left[F^{2}>2 \sigma\left(F^{2}\right)\right]=0.033$

$w R\left(F^{2}\right)=0.083$

$S=1.06$

3325 reflections

181 parameters

0 restraints

Primary atom site location: structure-invariant direct methods
$0.28 \times 0.13 \times 0.05 \mathrm{~mm}$

12258 measured reflections

3325 independent reflections

2839 reflections with $I>2 \sigma(I)$

$R_{\text {int }}=0.021$

$\theta_{\max }=28.4^{\circ}, \theta_{\min }=1.4^{\circ}$

$h=-18 \rightarrow 20$

$k=-8 \rightarrow 8$

$l=-19 \rightarrow 14$

Secondary atom site location: difference Fourier map

Hydrogen site location: inferred from neighbouring sites

$\mathrm{H}$-atom parameters constrained

$w=1 /\left[\sigma^{2}\left(F_{\mathrm{o}}^{2}\right)+(0.0306 P)^{2}+0.755 P\right]$

where $P=\left(F_{\mathrm{o}}^{2}+2 F_{\mathrm{c}}^{2}\right) / 3$

$(\Delta / \sigma)_{\max }=0.001$

$\Delta \rho_{\max }=0.37 \mathrm{e}^{-3}$

$\Delta \rho_{\min }=-0.25$ e $\AA^{-3}$

\section{Special details}

Geometry. All e.s.d.'s (except the e.s.d. in the dihedral angle between two 1.s. planes) are estimated using the full covariance matrix. The cell e.s.d.'s are taken into account individually in the estimation of e.s.d.'s in distances, angles and torsion angles; correlations between e.s.d.'s in cell parameters are only used when they are defined by crystal symmetry. An approximate (isotropic) treatment of cell e.s.d.'s is used for estimating e.s.d.'s involving 1.s. planes.

Refinement. Refinement of $F^{2}$ against ALL reflections. The weighted $R$-factor $w R$ and goodness of fit $S$ are based on $F^{2}$, conventional $R$-factors $R$ are based on $F$, with $F$ set to zero for negative $F^{2}$. The threshold expression of $F^{2}>\sigma\left(F^{2}\right)$ is used only for calculating $R$-factors(gt) etc. and is not relevant to the choice of reflections for refinement. $R$-factors based on $F^{2}$ are statistically about twice as large as those based on $F$, and $R$ - factors based on ALL data will be even larger.

Fractional atomic coordinates and isotropic or equivalent isotropic displacement parameters $\left(\AA^{2}\right)$

\begin{tabular}{lllll}
\hline & $x$ & $y$ & $z$ & $U_{\text {iso }} / U_{\text {eq }}$ \\
\hline C11 & $0.64583(2)$ & $0.83763(5)$ & $0.61691(2)$ & $0.02134(9)$ \\
O1 & $0.35931(6)$ & $0.24736(14)$ & $0.62698(7)$ & $0.0185(2)$ \\
O2 & $0.21611(7)$ & $0.17409(16)$ & $0.60995(8)$ & $0.0267(2)$ \\
C1 & $0.39949(9)$ & $0.6045(2)$ & $0.59464(9)$ & $0.0149(2)$ \\
C2 & $0.42529(9)$ & $0.3975(2)$ & $0.62766(9)$ & $0.0156(2)$ \\
C3 & $0.51713(9)$ & $0.3250(2)$ & $0.65950(9)$ & $0.0176(3)$ \\
H3A & 0.5325 & 0.1864 & 0.6827 & $0.021^{*}$ \\
C4 & $0.58581(9)$ & $0.4603(2)$ & $0.65657(9)$ & $0.0186(3)$ \\
H4A & 0.6477 & 0.4141 & 0.6777 & $0.022^{*}$ \\
C5 & $0.56024(9)$ & $0.6657(2)$ & $0.62152(9)$ & $0.0166(3)$ \\
C6 & $0.46877(9)$ & $0.7392(2)$ & $0.59102(9)$ & $0.0161(2)$ \\
H6A & 0.4536 & 0.8781 & 0.5682 & $0.019^{*}$ \\
C7 & $0.27232(9)$ & $0.3112(2)$ & $0.61886(10)$ & $0.0186(3)$ \\
C8 & $0.25822(9)$ & $0.5461(2)$ & $0.62445(10)$ & $0.0181(3)$
\end{tabular}




$\begin{array}{lllll}\text { H8A } & 0.2881 & 0.5907 & 0.6931 & 0.022^{*} \\ \text { H8B } & 0.1913 & 0.5753 & 0.5998 & 0.022^{*} \\ \text { C9 } & 0.29900(9) & 0.6771(2) & 0.56459(9) & 0.0158(2) \\ \text { H9A } & 0.2998 & 0.8275 & 0.5824 & 0.019^{*} \\ \text { C10 } & 0.24087(9) & 0.6545(2) & 0.45496(9) & 0.0163(2) \\ \text { H10A } & 0.2362 & 0.5208 & 0.4265 & 0.020^{*} \\ \text { C11 } & 0.19575(9) & 0.8170(2) & 0.39674(10) & 0.0170(3) \\ \text { H11A } & 0.2035 & 0.9496 & 0.4269 & 0.020^{*} \\ \text { C12 } & 0.13500(9) & 0.8094(2) & 0.28984(9) & 0.0158(2) \\ \text { C13 } & 0.07977(9) & 0.9877(2) & 0.24476(10) & 0.0189(3) \\ \text { H13A } & 0.0856 & 1.1106 & 0.2816 & 0.023^{*} \\ \text { C14 } & 0.01637(9) & 0.9834(2) & 0.14573(10) & 0.0213(3) \\ \text { H14A } & -0.0200 & 1.1029 & 0.1169 & 0.026^{*} \\ \text { C15 } & 0.00700(9) & 0.8019(2) & 0.08960(10) & 0.0209(3) \\ \text { H15A } & -0.0367 & 0.7976 & 0.0238 & 0.025^{*} \\ \text { C16 } & 0.06374(9) & 0.6256(2) & 0.13271(10) & 0.0202(3) \\ \text { H16A } & 0.0591 & 0.5048 & 0.0949 & 0.024^{*} \\ \text { C17 } & 0.12697(9) & 0.6293(2) & 0.23151(10) & 0.0184(3) \\ \text { H17A } & 0.1645 & 0.5109 & 0.2594 & 0.022^{*}\end{array}$

Atomic displacement parameters $\left(\AA^{2}\right)$

\begin{tabular}{lllllll}
\hline & $U^{11}$ & $U^{22}$ & $U^{33}$ & $U^{12}$ & $U^{13}$ & $U^{23}$ \\
\hline C11 & $0.01884(16)$ & $0.02132(17)$ & $0.02491(18)$ & $-0.00529(12)$ & $0.01029(13)$ & $-0.00125(13)$ \\
O1 & $0.0182(4)$ & $0.0133(4)$ & $0.0248(5)$ & $-0.0015(4)$ & $0.0099(4)$ & $0.0013(4)$ \\
O2 & $0.0231(5)$ & $0.0219(5)$ & $0.0368(6)$ & $-0.0055(4)$ & $0.0142(5)$ & $0.0006(4)$ \\
C1 & $0.0160(6)$ & $0.0155(6)$ & $0.0117(6)$ & $0.0004(5)$ & $0.0044(5)$ & $-0.0005(5)$ \\
C2 & $0.0172(6)$ & $0.0153(6)$ & $0.0138(6)$ & $-0.0027(5)$ & $0.0062(5)$ & $-0.0011(5)$ \\
C3 & $0.0196(6)$ & $0.0145(6)$ & $0.0169(6)$ & $0.0023(5)$ & $0.0061(5)$ & $0.0020(5)$ \\
C4 & $0.0156(6)$ & $0.0203(6)$ & $0.0180(6)$ & $0.0011(5)$ & $0.0053(5)$ & $-0.0005(5)$ \\
C5 & $0.0173(6)$ & $0.0174(6)$ & $0.0156(6)$ & $-0.0044(5)$ & $0.0073(5)$ & $-0.0019(5)$ \\
C6 & $0.0198(6)$ & $0.0136(6)$ & $0.0138(6)$ & $-0.0009(5)$ & $0.0060(5)$ & $0.0000(5)$ \\
C7 & $0.0188(6)$ & $0.0209(7)$ & $0.0168(6)$ & $-0.0015(5)$ & $0.0081(5)$ & $0.0003(5)$ \\
C8 & $0.0177(6)$ & $0.0191(6)$ & $0.0187(6)$ & $0.0002(5)$ & $0.0087(5)$ & $-0.0008(5)$ \\
C9 & $0.0160(6)$ & $0.0134(6)$ & $0.0169(6)$ & $0.0003(5)$ & $0.0059(5)$ & $-0.0006(5)$ \\
C10 & $0.0154(6)$ & $0.0153(6)$ & $0.0176(6)$ & $-0.0010(5)$ & $0.0064(5)$ & $-0.0020(5)$ \\
C11 & $0.0160(6)$ & $0.0166(6)$ & $0.0195(6)$ & $-0.0009(5)$ & $0.0087(5)$ & $-0.0008(5)$ \\
C12 & $0.0137(5)$ & $0.0173(6)$ & $0.0173(6)$ & $-0.0005(5)$ & $0.0076(5)$ & $0.0027(5)$ \\
C13 & $0.0211(6)$ & $0.0175(6)$ & $0.0214(7)$ & $0.0022(5)$ & $0.0123(5)$ & $0.0013(5)$ \\
C14 & $0.0206(6)$ & $0.0235(7)$ & $0.0218(7)$ & $0.0074(5)$ & $0.0110(5)$ & $0.0069(5)$ \\
C15 & $0.0170(6)$ & $0.0297(7)$ & $0.0158(6)$ & $0.0015(5)$ & $0.0067(5)$ & $0.0029(5)$ \\
C16 & $0.0200(6)$ & $0.0216(7)$ & $0.0201(7)$ & $-0.0018(5)$ & $0.0096(5)$ & $-0.0031(5)$ \\
C17 & $0.0176(6)$ & $0.0167(6)$ & $0.0217(7)$ & $0.0031(5)$ & $0.0092(5)$ & $0.0030(5)$ \\
& & & & & &
\end{tabular}

Geometric parameters $(\AA, \stackrel{\circ}{)})$

\begin{tabular}{llll}
\hline $\mathrm{C} 11-\mathrm{C} 5$ & $1.7456(13)$ & $\mathrm{C} 9-\mathrm{C} 10$ & $1.5049(17)$ \\
$\mathrm{O} 1-\mathrm{C} 7$ & $1.3758(15)$ & $\mathrm{C} 9-\mathrm{H} 9 \mathrm{~A}$ & 0.9800
\end{tabular}




\begin{tabular}{|c|c|c|c|}
\hline $\mathrm{O} 1-\mathrm{C} 2$ & $1.3962(15)$ & $\mathrm{C} 10-\mathrm{C} 11$ & $1.3322(18)$ \\
\hline $\mathrm{O} 2-\mathrm{C} 7$ & $1.1981(16)$ & $\mathrm{C} 10-\mathrm{H} 10 \mathrm{~A}$ & 0.9300 \\
\hline $\mathrm{C} 1-\mathrm{C} 2$ & $1.3880(18)$ & $\mathrm{C} 11-\mathrm{C} 12$ & $1.4722(18)$ \\
\hline $\mathrm{C} 1-\mathrm{C} 6$ & $1.3953(17)$ & $\mathrm{C} 11-\mathrm{H} 11 \mathrm{~A}$ & 0.9300 \\
\hline $\mathrm{C} 1-\mathrm{C} 9$ & $1.5127(17)$ & $\mathrm{C} 12-\mathrm{C} 13$ & $1.4002(18)$ \\
\hline $\mathrm{C} 2-\mathrm{C} 3$ & $1.3867(18)$ & $\mathrm{C} 12-\mathrm{C} 17$ & $1.4004(18)$ \\
\hline $\mathrm{C} 3-\mathrm{C} 4$ & $1.3863(18)$ & $\mathrm{C} 13-\mathrm{C} 14$ & $1.3884(19)$ \\
\hline $\mathrm{C} 3-\mathrm{H} 3 \mathrm{~A}$ & 0.9300 & $\mathrm{C} 13-\mathrm{H} 13 \mathrm{~A}$ & 0.9300 \\
\hline $\mathrm{C} 4-\mathrm{C} 5$ & $1.3861(19)$ & $\mathrm{C} 14-\mathrm{C} 15$ & $1.385(2)$ \\
\hline $\mathrm{C} 4-\mathrm{H} 4 \mathrm{~A}$ & 0.9300 & $\mathrm{C} 14-\mathrm{H} 14 \mathrm{~A}$ & 0.9300 \\
\hline $\mathrm{C} 5-\mathrm{C} 6$ & $1.3856(18)$ & $\mathrm{C} 15-\mathrm{C} 16$ & $1.3945(19)$ \\
\hline $\mathrm{C} 6-\mathrm{H} 6 \mathrm{~A}$ & 0.9300 & $\mathrm{C} 15-\mathrm{H} 15 \mathrm{~A}$ & 0.9300 \\
\hline $\mathrm{C} 7-\mathrm{C} 8$ & $1.4990(19)$ & $\mathrm{C} 16-\mathrm{C} 17$ & $1.3851(19)$ \\
\hline $\mathrm{C} 8-\mathrm{C} 9$ & $1.5394(17)$ & $\mathrm{C} 16-\mathrm{H} 16 \mathrm{~A}$ & 0.9300 \\
\hline $\mathrm{C} 8-\mathrm{H} 8 \mathrm{~A}$ & 0.9700 & C17-H17A & 0.9300 \\
\hline $\mathrm{C} 8-\mathrm{H} 8 \mathrm{~B}$ & 0.9700 & & \\
\hline $\mathrm{C} 7-\mathrm{O} 1-\mathrm{C} 2$ & $120.43(10)$ & $\mathrm{C} 10-\mathrm{C} 9-\mathrm{C} 8$ & $111.91(10)$ \\
\hline $\mathrm{C} 2-\mathrm{C} 1-\mathrm{C} 6$ & $117.87(11)$ & $\mathrm{C} 1-\mathrm{C} 9-\mathrm{C} 8$ & $107.64(10)$ \\
\hline $\mathrm{C} 2-\mathrm{C} 1-\mathrm{C} 9$ & $119.80(11)$ & $\mathrm{C} 10-\mathrm{C} 9-\mathrm{H} 9 \mathrm{~A}$ & 108.6 \\
\hline $\mathrm{C} 6-\mathrm{C} 1-\mathrm{C} 9$ & $122.32(11)$ & $\mathrm{C} 1-\mathrm{C} 9-\mathrm{H} 9 \mathrm{~A}$ & 108.6 \\
\hline $\mathrm{C} 3-\mathrm{C} 2-\mathrm{C} 1$ & $122.19(12)$ & $\mathrm{C} 8-\mathrm{C} 9-\mathrm{H} 9 \mathrm{~A}$ & 108.6 \\
\hline $\mathrm{C} 3-\mathrm{C} 2-\mathrm{O} 1$ & $115.96(11)$ & $\mathrm{C} 11-\mathrm{C} 10-\mathrm{C} 9$ & $123.12(12)$ \\
\hline $\mathrm{C} 1-\mathrm{C} 2-\mathrm{O} 1$ & $121.80(11)$ & $\mathrm{C} 11-\mathrm{C} 10-\mathrm{H} 10 \mathrm{~A}$ & 118.4 \\
\hline $\mathrm{C} 4-\mathrm{C} 3-\mathrm{C} 2$ & $119.62(12)$ & $\mathrm{C} 9-\mathrm{C} 10-\mathrm{H} 10 \mathrm{~A}$ & 118.4 \\
\hline $\mathrm{C} 4-\mathrm{C} 3-\mathrm{H} 3 \mathrm{~A}$ & 120.2 & $\mathrm{C} 10-\mathrm{C} 11-\mathrm{C} 12$ & $127.08(12)$ \\
\hline $\mathrm{C} 2-\mathrm{C} 3-\mathrm{H} 3 \mathrm{~A}$ & 120.2 & $\mathrm{C} 10-\mathrm{C} 11-\mathrm{H} 11 \mathrm{~A}$ & 116.5 \\
\hline $\mathrm{C} 5-\mathrm{C} 4-\mathrm{C} 3$ & $118.59(12)$ & $\mathrm{C} 12-\mathrm{C} 11-\mathrm{H} 11 \mathrm{~A}$ & 116.5 \\
\hline $\mathrm{C} 5-\mathrm{C} 4-\mathrm{H} 4 \mathrm{~A}$ & 120.7 & $\mathrm{C} 13-\mathrm{C} 12-\mathrm{C} 17$ & $118.20(12)$ \\
\hline $\mathrm{C} 3-\mathrm{C} 4-\mathrm{H} 4 \mathrm{~A}$ & 120.7 & $\mathrm{C} 13-\mathrm{C} 12-\mathrm{C} 11$ & $118.61(12)$ \\
\hline $\mathrm{C} 6-\mathrm{C} 5-\mathrm{C} 4$ & $121.80(12)$ & $\mathrm{C} 17-\mathrm{C} 12-\mathrm{C} 11$ & $123.15(12)$ \\
\hline $\mathrm{C} 6-\mathrm{C} 5-\mathrm{Cl} 1$ & $119.05(10)$ & $\mathrm{C} 14-\mathrm{C} 13-\mathrm{C} 12$ & $120.90(12)$ \\
\hline $\mathrm{C} 4-\mathrm{C} 5-\mathrm{Cl} 1$ & $119.14(10)$ & $\mathrm{C} 14-\mathrm{C} 13-\mathrm{H} 13 \mathrm{~A}$ & 119.6 \\
\hline $\mathrm{C} 5-\mathrm{C} 6-\mathrm{C} 1$ & $119.89(12)$ & $\mathrm{C} 12-\mathrm{C} 13-\mathrm{H} 13 \mathrm{~A}$ & 119.6 \\
\hline $\mathrm{C} 5-\mathrm{C} 6-\mathrm{H} 6 \mathrm{~A}$ & 120.1 & $\mathrm{C} 15-\mathrm{C} 14-\mathrm{C} 13$ & $120.31(13)$ \\
\hline $\mathrm{C} 1-\mathrm{C} 6-\mathrm{H} 6 \mathrm{~A}$ & 120.1 & $\mathrm{C} 15-\mathrm{C} 14-\mathrm{H} 14 \mathrm{~A}$ & 119.8 \\
\hline $\mathrm{O} 2-\mathrm{C} 7-\mathrm{O} 1$ & $117.02(12)$ & $\mathrm{C} 13-\mathrm{C} 14-\mathrm{H} 14 \mathrm{~A}$ & 119.8 \\
\hline $\mathrm{O} 2-\mathrm{C} 7-\mathrm{C} 8$ & $126.49(12)$ & $\mathrm{C} 14-\mathrm{C} 15-\mathrm{C} 16$ & $119.39(13)$ \\
\hline $\mathrm{O} 1-\mathrm{C} 7-\mathrm{C} 8$ & $116.47(11)$ & $\mathrm{C} 14-\mathrm{C} 15-\mathrm{H} 15 \mathrm{~A}$ & 120.3 \\
\hline $\mathrm{C} 7-\mathrm{C} 8-\mathrm{C} 9$ & $112.71(10)$ & $\mathrm{C} 16-\mathrm{C} 15-\mathrm{H} 15 \mathrm{~A}$ & 120.3 \\
\hline $\mathrm{C} 7-\mathrm{C} 8-\mathrm{H} 8 \mathrm{~A}$ & 109.0 & $\mathrm{C} 17-\mathrm{C} 16-\mathrm{C} 15$ & $120.39(12)$ \\
\hline $\mathrm{C} 9-\mathrm{C} 8-\mathrm{H} 8 \mathrm{~A}$ & 109.0 & $\mathrm{C} 17-\mathrm{C} 16-\mathrm{H} 16 \mathrm{~A}$ & 119.8 \\
\hline $\mathrm{C} 7-\mathrm{C} 8-\mathrm{H} 8 \mathrm{~B}$ & 109.0 & $\mathrm{C} 15-\mathrm{C} 16-\mathrm{H} 16 \mathrm{~A}$ & 119.8 \\
\hline $\mathrm{C} 9-\mathrm{C} 8-\mathrm{H} 8 \mathrm{~B}$ & 109.0 & $\mathrm{C} 16-\mathrm{C} 17-\mathrm{C} 12$ & $120.75(12)$ \\
\hline $\mathrm{H} 8 \mathrm{~A}-\mathrm{C} 8-\mathrm{H} 8 \mathrm{~B}$ & 107.8 & $\mathrm{C} 16-\mathrm{C} 17-\mathrm{H} 17 \mathrm{~A}$ & 119.6 \\
\hline $\mathrm{C} 10-\mathrm{C} 9-\mathrm{C} 1$ & $111.54(10)$ & $\mathrm{C} 12-\mathrm{C} 17-\mathrm{H} 17 \mathrm{~A}$ & 119.6 \\
\hline $\mathrm{C} 6-\mathrm{C} 1-\mathrm{C} 2-\mathrm{C} 3$ & $2.07(19)$ & $\mathrm{C} 2-\mathrm{C} 1-\mathrm{C} 9-\mathrm{C} 10$ & $-94.72(14)$ \\
\hline
\end{tabular}




$$
\begin{aligned}
& \mathrm{C} 9-\mathrm{C} 1-\mathrm{C} 2-\mathrm{C} 3 \\
& \mathrm{C} 6-\mathrm{C} 1-\mathrm{C} 2-\mathrm{O} 1 \\
& \mathrm{C} 9-\mathrm{C} 1-\mathrm{C} 2-\mathrm{O} 1 \\
& \mathrm{C} 7-\mathrm{O} 1-\mathrm{C} 2-\mathrm{C} 3 \\
& \mathrm{C} 7-\mathrm{O} 1-\mathrm{C} 2-\mathrm{C} 1 \\
& \mathrm{C} 1-\mathrm{C} 2-\mathrm{C} 3-\mathrm{C} 4 \\
& \mathrm{O} 1-\mathrm{C} 2-\mathrm{C} 3-\mathrm{C} 4 \\
& \mathrm{C} 2-\mathrm{C} 3-\mathrm{C} 4-\mathrm{C} 5 \\
& \mathrm{C} 3-\mathrm{C} 4-\mathrm{C} 5-\mathrm{C} 6 \\
& \mathrm{C} 3-\mathrm{C} 4-\mathrm{C} 5-\mathrm{C} 1 \\
& \mathrm{C} 4-\mathrm{C} 5-\mathrm{C} 6-\mathrm{C} 1 \\
& \mathrm{C} 11-\mathrm{C} 5-\mathrm{C} 6-\mathrm{C} 1 \\
& \mathrm{C} 2-\mathrm{C} 1-\mathrm{C} 6-\mathrm{C} 5 \\
& \mathrm{C} 9-\mathrm{C} 1-\mathrm{C} 6-\mathrm{C} 5 \\
& \mathrm{C} 2-\mathrm{O} 1-\mathrm{C} 7-\mathrm{O} 2 \\
& \mathrm{C} 2-\mathrm{O} 1-\mathrm{C} 7-\mathrm{C} 8 \\
& \mathrm{O} 2-\mathrm{C} 7-\mathrm{C} 8-\mathrm{C} 9 \\
& \mathrm{O} 1-\mathrm{C} 7-\mathrm{C} 8-\mathrm{C} 9
\end{aligned}
$$

$-177.61(12)$

$-175.28(11)$

$5.04(18)$

$165.08(11)$

$-17.41(17)$

$-1.5(2)$

$175.98(11)$

$-0.02(19)$

$0.96(19)$

$-179.75(10)$

-0.38 (19)

$-179.67(9)$

$-1.11(18)$

$178.56(11)$

$173.59(12)$

$-7.79(17)$

$-138.65(14)$

42.87 (16)

$$
\begin{aligned}
& \mathrm{C} 6-\mathrm{C} 1-\mathrm{C} 9-\mathrm{C} 10 \\
& \mathrm{C} 2-\mathrm{C} 1-\mathrm{C} 9-\mathrm{C} 8 \\
& \mathrm{C} 6-\mathrm{C} 1-\mathrm{C} 9-\mathrm{C} 8 \\
& \mathrm{C} 7-\mathrm{C} 8-\mathrm{C} 9-\mathrm{C} 10 \\
& \mathrm{C} 7-\mathrm{C} 8-\mathrm{C} 9-\mathrm{C} 1 \\
& \mathrm{C} 1-\mathrm{C} 9-\mathrm{C} 10-\mathrm{C} 11 \\
& \mathrm{C} 8-\mathrm{C} 9-\mathrm{C} 10-\mathrm{C} 11 \\
& \mathrm{C} 9-\mathrm{C} 10-\mathrm{C} 11-\mathrm{C} 12 \\
& \mathrm{C} 10-\mathrm{C} 11-\mathrm{C} 12-\mathrm{C} 13 \\
& \mathrm{C} 10-\mathrm{C} 11-\mathrm{C} 12-\mathrm{C} 17 \\
& \mathrm{C} 17-\mathrm{C} 12-\mathrm{C} 13-\mathrm{C} 14 \\
& \mathrm{C} 11-\mathrm{C} 12-\mathrm{C} 13-\mathrm{C} 14 \\
& \mathrm{C} 12-\mathrm{C} 13-\mathrm{C} 14-\mathrm{C} 15 \\
& \mathrm{C} 13-\mathrm{C} 14-\mathrm{C} 15-\mathrm{C} 16 \\
& \mathrm{C} 14-\mathrm{C} 15-\mathrm{C} 16-\mathrm{C} 17 \\
& \mathrm{C} 15-\mathrm{C} 16-\mathrm{C} 17-\mathrm{C} 12 \\
& \mathrm{C} 13-\mathrm{C} 12-\mathrm{C} 17-\mathrm{C} 16 \\
& \mathrm{C} 11-\mathrm{C} 12-\mathrm{C} 17-\mathrm{C} 16
\end{aligned}
$$

$$
\begin{aligned}
& 85.61(14) \\
& 28.41(15) \\
& -151.26(12) \\
& 72.17(14) \\
& -50.73(14) \\
& -122.70(13) \\
& 116.65(13) \\
& -177.86(11) \\
& 168.28(12) \\
& -9.2(2) \\
& 2.21(18) \\
& -175.41(11) \\
& -0.25(19) \\
& -1.82(19) \\
& 1.90(19) \\
& 0.10(19) \\
& -2.13(18) \\
& 175.38(12)
\end{aligned}
$$

